Instituto Internacional de Investigación y Desarrollo Tecnológico Educativo INDTEC, C.A.

DOI: https://doi.org/10.29394/scientific.issn.2542-2987.2017.2.4.16.283-301

OAI-PMH: http://www.indteca.com/ojs/index.php/Revista Scientific/oai

\title{
Estrategias Instruccionales Alternativa para la Comprensión Lectora
}

\author{
Autora: Yajaira del Valle Cadenas Terán \\ Universidad Nacional Experimental "Rafael María Baralt", UNERMB \\ yajairaf@yahoo.com \\ Trujillo, Venezuela
}

\section{Resumen}

El propósito de esta investigación es exponer de manera significativa alternativas instruccionales- estratégicas que contribuyan a mejorar el proceso de lectura en los estudiantes universitarios para que se formen de manera integral, capaces de tomar decisiones críticas, reflexivas y exitosas en el campo académico. Las estrategias aplicadas aisladas al hecho educativo no producen ningún cambio, es necesario, que se planifiquen y se ejecuten en el contexto propio de la necesidad, para garantizar en cierta medida el éxito instruccional. También es fundamental que el docente sea el primero en apropiarse de ella. Este estudio se realizó con una revisión bibliográfica que sirve de fundamento instruccional - estratégico. Como conclusión se determinó la importancia de las estrategias instruccionales en la comprensión lectora, puesto que incrementan las habilidades comunicativas, proporcionan experiencias concretas o complejas y favorecen el aprendizaje significativo.

Palabras clave: estrategias instruccionales; aprendizaje significativo; comprensión lectora. 


\section{Instructional Strategies Alternative for Reading Comprehension}

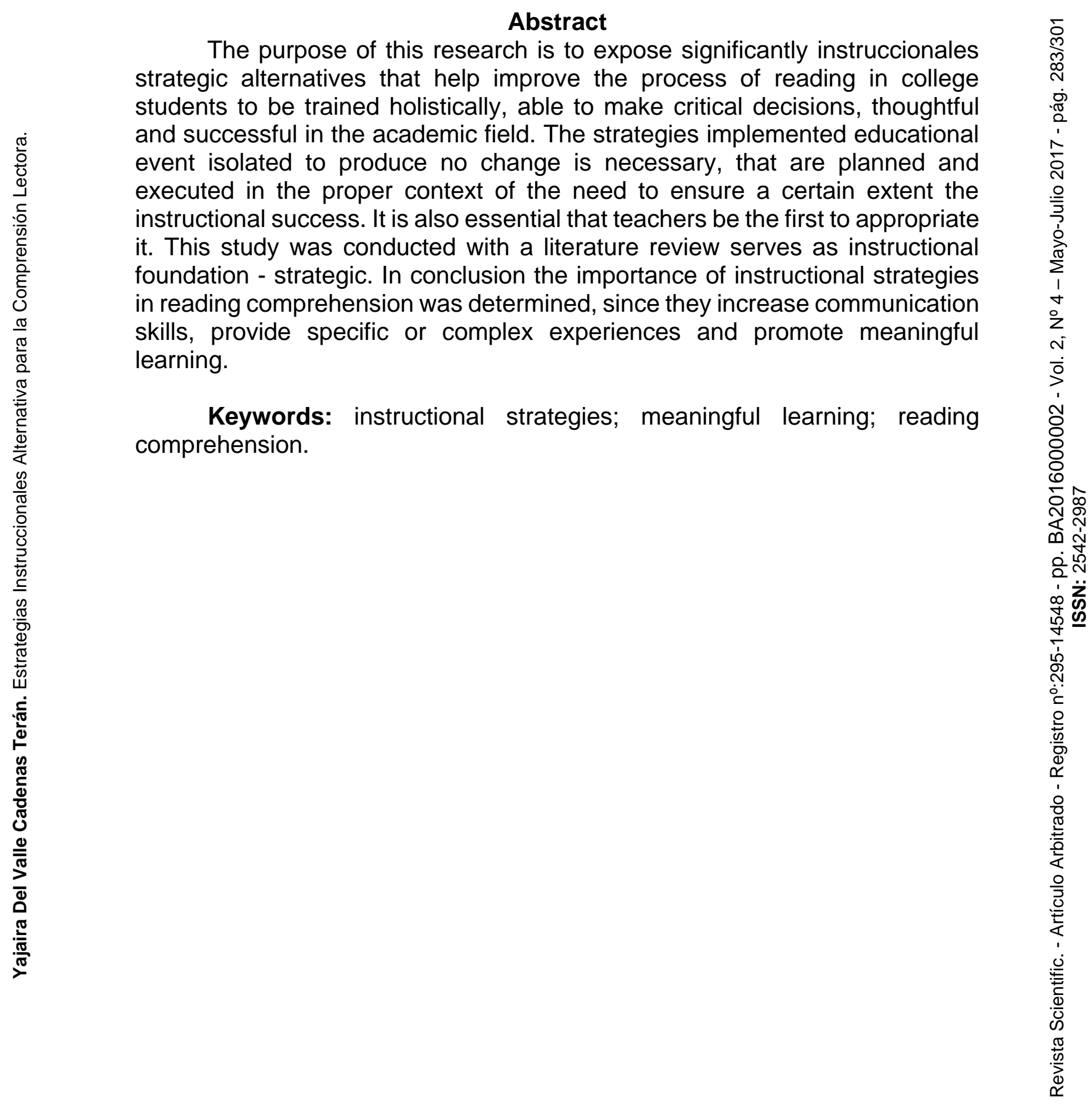

Date Received: 31-10-2016

Date Acceptance: 12-12-2016 


\section{Introducción}

El lenguaje es fundamental en toda actividad humana. Desde que el hombre nace se comunica con las demás personas que están a su alrededor. A medida que crece nutre los gestos en palabras, luego en oraciones, hasta que construye párrafos y forma su propio criterio del mundo que lo rodea. Por tanto, el lenguaje forma un papel importante en la evolución cognitiva, que hace se diferencie de los animales.

Cada día como ser que reflexiona debe enriquecer sus conocimientos y su léxico para comunicarse con mayor fluidez y precisión y la mejor forma de hacerlo es a través de la lectura. Goodman, (1982) citado en Sánchez, (2010) "Leer es producir significados. No obstante, es posible distinguir algunos tipos de lectura de acuerdo con el propósito que se persigue, el grado o nivel de comprensión, el tipo de texto y las técnicas aplicadas" (pág. 3).

Esto significa que leer, no es una actividad pasiva, es involucrarse, construir el significado del texto, es darle vida a un montón de palabras. La lectura es una actividad compleja, ya que influyen las experiencias pasadas, los conocimientos y cultura en general del lector e incluso los estados de ánimo que hace obtener resultados diferentes a la de los demás.

En este sentido, para la educación moral y profesional del estudiante González, (2003a) asegura que es "un proceso permanente, continuo y gradual en el ejercicio de la docencia, que implica la reflexión crítica y comprometida del profesor con la transformación de la práctica educativa, la calidad de su desempeño, en un ambiente dialógico y participativo..." (pág. 3).

En el ámbito internacional, nacional y local se plantea el problema de que los estudiantes de educación universitaria poseen bajo rendimiento porque no saben leer y escribir adecuadamente, no han desarrollado habilidades en comprensión lectora que le permitan un mejor desempeño como entes productivos dentro de la sociedad. 
Por ello, el docente debe aplicar estrategias instruccionales antes, en el momento y después del proceso de aprendizaje para que mejore la calidad de la educación, a fin de formar individuos capaces se enfrenten a cualquier situación o necesidad que se les presente y ocupe un lugar digno como miembro de la sociedad, donde se desenvuelve. De hecho, el docente debe enseñar estrategias para que el alumno lea comprensivamente, para que utilice la lectura como medio para aprender.

Para ello, es necesario que se sientan motivados y que dispongan de los medios adecuados para leer, progresivamente dirigir, controlar su aprendizaje y aplicar estrategias a situaciones distintas a las académicas. Por esa razón, surge esta investigación para exponer de forma teórica las estrategias instruccionales que proponen algunos autores y contribuyen al desarrollo de la comprensión lectora de los estudiantes universitarios. Para lograr este propósito el estudio se estructurará de la siguiente manera: el problema, bases teóricas, conclusiones y referencias.

\section{EI Problema}

El interés por la comprensión lectora no es nuevo, se han realizado estudios sobre la importancia de la lectura y se han ocupado de determinar lo que sucede cuando un lector comprende el texto. Actualmente, se ha intensificado este interés por el proceso de la comprensión lectora y los resultados que se han obtenido son semejantes de hace años atrás, porque se siguen utilizando estrategias o métodos arbitrarios para la enseñanza de la lectura. Por ejemplo, Aguirre, (2007) dice "se ha orientado a transformar el uso de la lengua escrita en estudiantes universitarios, buscando que mejoren sus modos de escribir, que lean textos de formas diversas y que practiquen la manera de revisar sus propios textos" (pág. 88) O sea, la lectura ha estado durante muchos años en el limbo del sistema educativo, y es la experiencia más importante para el aprendizaje y el rendimiento escolar. 
Por otro lado, Reimers, (2000) explica "el docente tiene uno de los desafíos más importantes de la educación latinoamericana formar ciudadanos capaces de pensar, de valorar su libertad y la de los demás y que tengan habilidades para hacerse cargo de mejorar su vida y la de sus familias" (pág. 23). Se piensa entonces, sea necesario que el individuo aprenda destrezas básicas, complejas necesarias para vivir en el siglo XXI, tales como: trabajar en grupo, resolver problemas, comunicarse efectivamente, negociar diferencias y especialmente a aprender a ejercer ciudadanía responsable, solidaria para vivir en democracia.

Por consiguiente, la educación debe ser una esperanza al progreso personal y profesional para el individuo, que contribuya a formar individuos competentes. Es importante enseñar a los niños a pensar, no sólo a aprender contenidos, sino a ser críticos y reflexivos. Esto requiere que los docentes enseñen con ejemplo, tratándose entre sí y a sus estudiantes con respeto, aceptando los puntos de vista de los demás, mediando para que éstos se traten con aprecio.

Al respecto, Cifuentes, (2004) sostiene "las causas de la mala lectura se debe a los viejos paradigmas con los que se les enseñó a leer, en Latinoamérica los jóvenes no saben leer, ya que no explican lo que leen y menos producen textos" (pág. 110). Es decir, leer eficientemente es una ventaja muy competitiva en un mundo en el que cada vez no basta un título universitario, sino se hace necesario un vasto cúmulo de conocimientos que es infinito e inabarcable.

En Venezuela, la falta de comprensión lectora se manifiesta en todos los niveles y modalidades de educación. Por ejemplo, en Educación Básica existen deficiencias desde la lectura literal hasta la lectura comprensiva. En Educación Media General y Técnica las dificultades en comprensión lectora ocupan un problema en la adquisición del aprendizaje, situación que se evidencia en el ámbito universitario. En este último nivel se suma la apatía de 
los estudiantes para realizar actividades de lectura, trayendo como consecuencia un bajo rendimiento académico. Una de las causas de este problema se centra en las estrategias que utilizan los docentes para facilitar la interiorización del aprendizaje.

Según, Dubois, (2002) "la mayoría de las universidades siguen aferradas a viejos modelos de enseñanza, donde reina la disertación, la transmisión de información, la repetición del saber, en lugar, del diálogo, la construcción de conocimientos, la interdisciplinariedad, el cuestionamiento y la reflexión" (pág. 98). En otras palabras, una institución educativa que posea estas características no puede formar al futuro profesional como persona crítica y creativa, todo lo contrario, los convierte en personas dependientes del saber de otros, sin capacidad para diferir, discernir o evaluar.

No obstante, el sistema educativo universitario está enmarcado en la concepción de la enseñanza transmisora. En las aulas de clase el alumno es un ser dependiente, cuyo rol es la búsqueda y memorización de conocimientos, ya que se espera que el profesor le proporcione toda la información. Como lo afirma Aguirre, (ob.cit.), "Ios alumnos leen sólo para estudiar, la lectura es un descifrado correcto y .la comprensión va asociada a la correcta oralización del texto" (pág. 33). De ahí que, la práctica de lectores y escritores se reduzca a la vida académica.

Desde este contexto, López, (2008), señala "las razones `por las cuales en Venezuela existe un bajo hábito de lectura son: el alto costo de los libros, y el auge de los medios audiovisuales y tecnológicos., pero, sobre todo, la educación familiar y el escaso incentivo en la escuela". Estos últimos son factores que inciden de manera conjunta o por separado en la tendencia del venezolano a no sentir motivación hacia la lectura. El trabajo de aula ha ido transformando la lectura en una actividad poco placentera y extremadamente escolarizada que no beneficia a la construcción del aprendizaje. 
Al respecto, Villamizar (2006) señala que la lectura "se trata de una actividad mental de nivel elevado que exige la adquisición no sólo de automatismos, sino también de posibilidades, de medios de decodificación del significado" (pág. 21). Esto implica que la lectura permite establecer nexos entre el lector y el autor del texto esenciales para el desarrollo individual y social del lector. Es decir, aprender a leer enlaza el desarrollo de estrategias para obtener sentido del texto, aparte de su estructura y del propósito del lector en el momento de leer.

Ello hace necesario, elevar la calidad de la educación de los estudiantes universitarios a objeto de formar profesionales capaces de analizar, resumir, criticar, y, sobre todo, a actuar, para ello, es necesario que desde el primer semestre se mantenga en contacto con la lectura para desarrollar habilidades en comprensión lectora.

Por los planteamientos descritos el propósito de este estudio es presentar una serie de estrategias instruccionales que sirvan de guía a los docentes de cualquier área, en el ámbito universitario, para que los estudiantes consideren la lectura como un proceso de comprensión. En este sentido, esta investigación teórica se propone responder la siguiente interrogante ¿Cuáles estrategas instruccionales contribuyen al desarrollo de la comprensión lectora en los estudiantes universitarios?

\subsection{Bases teóricas}

\subsubsection{Teorías que Sustentan la Investigación}

Actualmente, el docente tiene la necesidad de educar y formar alumnos que respondan a una sociedad cambiante, donde existe una creciente demanda social. Según Revilla, (2003) "el aprendizaje escolar no es mera acumulación de conocimientos, más bien, es una integración de conocimientos nuevos con los previos para que modifique la organización de éstos últimos" (pág. 74). Para que el participante logre este proceso necesita ciertas 
estrategias que faciliten la adquisición, el almacenamiento y la utilización de la información.

Por ello, el quehacer educativo no debe permanecer aferrado a ciertos paradigmas, debe aceptar nuevas perspectivas para mejores logros y satisfacciones de los que integran este hecho. Heller, (1996) explica "todos los involucrados en el hecho educativo deben poseer nuevas visiones, que aumente las capacidades para explorar, descubrir y aplicar conocimientos en una realidad de complejidades y caos, que reclama el rescate de valores y el crecimiento permanente" (pág. 22). Así el desafío del docente es repensar en el proceso educativo, educando desde una visión holística del hombre, desarrollando su potencial.

En este orden de ideas, el constructivismo es una teoría que sustenta el estudio esencialmente un enfoque epistemológico-lógico, que sostiene que todo conocimiento es construido como resultado de procesos cognitivos dentro de la mente humana. En este sentido, rechaza la idea de que el conocimiento sea la representación de la realidad externa independiente del espectador. Al analizar la teoría constructivista en el proceso de aprendizaje, los docentes como los participantes deben ser conscientes de su visión del mundo.

De ahí que, el docente constructivista integra al estudiante en la planificación, estrategias, objetivos, recursos y toma de decisiones referente al proceso de aprendizaje. Ambos, cumplen roles activos para el logro de los objetivos propuestos para mejorar la participación, incrementar la responsabilidad, implementando el trabajo colaborativo. Por tanto, a partir del constructivismo, surgen nuevos postulados: como la Teoría Sociocultural de Lev Vygotsky (1987).

Desde el punto de vista constructivista, para Vygotsky, la construcción y reconstrucción del conocimiento, es el producto de las interrelaciones sociales de la comunicación y de la actividad interpretada como mediación a través del uso de instrumentos y estrategias que permiten la regulación y la 
trasformación del mundo externo y del propio desempeño humano. Es decir, las funciones psíquicas superiores son el resultado del desarrollo sociocultural y no biológico y se adquieren a través de la internalización de instrumentos como el lenguaje como el más predominante porque proporciona los agentes culturales.

En este sentido, Vygotsky, (ob.cit.) argumenta: "las relaciones entre funciones psíquicas superiores fueron alguna vez relaciones reales entre agentes" (pág. 158). Para este autor, el lenguaje es la herramienta que regula el pensamiento, el alumno adquiere nuevas visiones culturales que son necesarias para la participación social. De esta manera se puede considerar que para la teoría Vigotskyana "el docente es un mediador en el aprendizaje debe propiciar situaciones desde el punto de vista cognitivo y social para orientar al alumno en el proceso. Por tanto, esta teoría puede transferirse al proceso de enseñanza actual y más a mejorar la comprensión lectora, ya que invita al docente a aplicar estrategias instruccionales que favorezca el trabajo cooperativo y la comunicación para el desarrollo del aprendizaje significativo.

Otro sustento teórico, para el estudio es la Teoría del Aprendizaje Significativo de Ausubel, (1988) citado en Mata y Ávila, (2012) establecen "como una adquisición de significados nuevos; presume una tendencia al aprendizaje significativo y una tarea de aprendizaje potencialmente significativo" (pág. 377). Esta teoría está sustentada en dos categorías, que son: cognitiva referida a los factores internos del alumno y motivacional que abarca los factores situacionales del aprendizaje. El autor considera la categoría cognitiva como la más importante para lograr el aprendizaje significativo, sustentada en que los conocimientos que posee el alumno en su estructura cognitiva es lo que permite la asimilación de nuevos conocimientos, mientras que la categoría motivacional, a pesar de considerarla en segundo plano, contribuyen en el proceso. 
Por lo tanto, el docente debe incentivar en el participante, la incorporación de nuevas ideas y conceptos a su estructura cognitiva, debe utilizar estrategias adecuadas para que el alumno desarrolle su capacidad para el aprendizaje y logre los objetivos propuestos, ejecutando acciones de forma intencional, pero no arbitraria, entre significados previos y conocimientos nuevos. Por otra parte, Ausubel (ob.cit.) explica: "se aprende a leer percibiendo el significado potencial de mensajes escritos y luego relacionar el significado percibido con la estructura cognitiva a fin de comprenderlo" (pág. 54). Es decir, significa, que se comparan los símbolos escritos nuevos con los símbolos hablados, que ya son familiares y significativos.

\subsection{Bases Conceptuales}

\subsubsection{Comprensión Lectora}

La comprensión lectora es la habilidad que posee el lector para entender la información del texto. Como afirma, Garza \& Leventhal, (2003) "la comprensión lectora es un proceso activo, que involucra factores internos (escritor, texto y entorno) y externos (cantidad y calidad de conocimientos 'básicos referente al uso de la lengua y el mundo)" (pág. 38).

Al respecto, este autor considera cuatro niveles de comprensión lectora: (a) Literal: consiste en poner en práctica los procesos básicos del pensamiento y en la aplicación de esquemas mentales para el logro de la representación de la información dada en los textos; (b) Inferencial: es cuando el lector tiene la habilidad de obtener datos a partir de lo leído, obteniendo sus propias conclusiones; (c) Analógico: que se refiere cuando el lector utiliza la información previa que se ha obtenido a través de las lecturas y (d) Crítico: es el último nivel: en donde el lector ha desarrollado habilidades lectoras y es más analítico, porque codifica las ideas ye interpreta la información, valorando el texto desde su juicio crítico. 


\subsubsection{Estrategias Instruccionales}

La enseñanza de la lectura en las aulas de clase exige un docente innovador y una institución que este abierta a realizar actividades de aprendizaje como un todo armónico, que relacione, lo didáctico con lo creativo, cuyo fin es que los alumnos aprendan entreteniéndose. Autores como Rosemblatt (1975), Santaella, (1986), Navas (1995), citados en Puerta (2000) han realizado investigaciones para explicar los problemas de la lectura como un proceso activo de construcción de conocimientos. Ellos han resaltado primordialmente el rol del docente y de las instituciones educativas como entes sensibilizadores y promotores de la lectura.

En su rol de mediador, el docente está encargado de incentivar a los alumnos en una "aventura que englobe la animación por la lectura, logrando la construcción de sus propios aprendizajes" (Puerta). Para ello, se acompañará de sensibilidad, conocimientos de la lectura que organiza estrategias de enseñanza que apoyen su trabajo y que se adecuen a las necesidades e intereses de los alumnos" (pág. 168).

Las estrategias de la enseñanza se entienden como procedimientos que utiliza el docente para ayudar al alumno a construir sus propios aprendizajes; para Monereo, (2004) define a las estrategias de aprendizaje como "procesos de toma de decisiones en los cuales el alumno elige y recupera, los conocimientos que necesita para un determinado objetivo, dependiendo de las características de la situación educativa en que se produce la acción" (pág. 27). Por ello, las estrategias de aprendizaje han de concebirse como los medios que un estudiante emplea en forma consciente, controlada e intencional como instrumentos dúctiles para aprender y solucionar problemas.

Por lo tanto, en el proceso de la lectura, las estrategias de enseñanza son herramientas que utiliza el docente para que el estudiante logre comprender la información contenida en el texto para transferirlas a situaciones posteriores. Desde esta temática, Díaz y Hernández, (2002) 
presentan estrategias de enseñanza que el docente puede emplear para facilitar el aprendizaje significativo (pág. 17):

1). Las estrategias preinstruccionales "son las que se utilizan al inicio del proceso ayudan a activar o generar los conocimientos y experiencias previas de los estudiantes y establecer expectativas referentes al tema que se va a estudiar". Estas estrategias permiten que el docente conozca lo que ya saben que los alumnos y utilicen esos conocimientos para promover nuevos aprendizajes. Algunas de estas estrategias: objetivos o propósitos del aprendizaje, organizadores previos y lluvia de ideas.

2). Las estrategias coinstruccionales "son las que ayudan a desarrollar los contenidos curriculares durante el proceso de enseñanza o de la lectura del texto. Facilitan la detención de información como: conceptos, organización, estructura y relaciones de los contenidos, además mantienen la atención y motivación del alumno". Entre estas estrategias están: ilustraciones, preguntas intercaladas, analogías, mapas conceptuales y uso de estructuras textuales.

3). Las estrategias post-institucionales: son "las que se presentan después del desarrollo del contenido que el alumno aprende. Ayudan al alumno valorar su propio aprendizaje, ya que puede integrar, sintetizar y criticar el material estudiado". Entre estas estrategias están: resúmenes, mapas conceptuales y redes semánticas.

En ese orden de ideas, se describen las estrategias más utilizadas antes, durante y después del proceso de enseñanza:

a). Objetivos o intenciones de la lectura: corresponde a los enunciados que describen las actividades de aprendizaje sobre el texto que se va a leer y los efectos que se pretenden conseguir en los alumnos en finalizar la lectura. Antes de iniciar la lectura, el docente como mediador del proceso debe incentivar a los alumnos a formular sus objetivos. 
b). Ilustraciones: constituyen estrategias muy utilizadas en el proceso de enseñanza pueden llamar o distraer la atención del lector. Estas son todos los esquemas, gráficos y fotografías que se utilizan para comunicar ideas de forma concreta, conceptos de forma visual o instrucciones procedimentales. Las funciones que emplea en un texto son de dirigir la atención de los alumnos, permitir la explicación en términos visuales lo que es difícil comunicar de forma verbal, favorecer la retención de información y clarificar e integrar la información en un todo.

c). Resumen: esta estrategia es frecuente en todas las actividades. Es una versión breve del contenido que habrá de aprenderse, donde se enfatiza los puntos sobresalientes de la información. El resumen es una macro estructura del discurso oral o escrito, es decir, es una síntesis del texto leído. Las funciones son ubicar al estudiante dentro de la estructura global del texto, identificar la información importante, organizar, integrar, y consolidar la información que el alumno ha adquirido, facilitar el aprendizaje y familiarizar el contenido.

d). Organizadores Previos: esta es una estrategia puede elaborarse en forma de prosa en verso o en forma de mapas, y gráficos conceptos diagramados permitiendo visualizar sus relaciones esenciales y constituye el material introductorio compuesto por un conjunto de conceptos $y$ preposiciones de mayor nivel de inclusión y generalidad que la información nueva que los estudiantes deban aprender.

e). Preguntas intercaladas: estas son aquellas que el alumno se platea a medida que va leyendo, facilitando el proceso de aprendizaje. Son preguntas que se van insertando en partes importantes del texto, en cada determinado de números de secciones o párrafos. El número de párrafos y de preguntas no están establecidas, dependerá del docente, pero deben planificarse de forma proporcionada para no abrumar al lector. 
Las preguntas intercaladas evalúan los aspectos: adquisición de conocimientos, comprensión y la aplicación de los contenidos aprendidos, además ayudan a monitorear el avance del estudiante. Las funciones de esta estrategia son: mantener la atención del estudiante a lo largo del estudio del material, favorecer la práctica y la reflexión sobre la información que se ha de aprender y el aprendizaje significativo del contenido.

f). Analogías: esta estrategia se emplea cuando la información que se ha de aprender se relaciona con conocimientos ya aprendidos que el estudiante maneja sin ninguna dificultad. Por el contrario, si el estudiante relaciona la información nueva con datos menos comprensibles solo se confundirá más. Se compone de cuatro elementos: el contenido abstracto y complejo que el estudiante debe aprender, el vehículo que es el contenido familiar y concreto que el estudiante utilizará para establecer la analogía, el conectivo que conecta al contenido y el vehículo (se parece a, "puede ser comparado con"), y la explicación de la relación analógica que debe poseer un límite. Entre las funciones de esta estrategia esta: incrementar para preparar al estudiante a experiencias complejas, favorecer el aprendizaje significativo familiarizando al estudiante a informaciones concreta y mejorar la comprensión de contenidos complejos.

g). Pistas tipográficas: ellas constituyen avisos que se dan en el texto para organizar y enfatizar algunos elementos de la información contenida. Entre ellas están: manejo alternado de mayúscula y minúsculas, uso de distintos tipo y tamaño de letras, empleo de títulos y subtítulos, subrayado de contenidos principales, empleo de logotipos, manejo de distintos colores en el texto La función esencial de esta estrategia es ayudar al estudiante en la detención en los elementos más importantes del texto para obtener una idea global de la organización e interrelación de estos elementos.

h). Mapas conceptuales: esta estrategia sirve para jerarquizar los conceptos más importantes de un texto, está formado por conceptos, 
proposiciones y palabras de enlace. Lo define como representación graficas de segmentos de información o conocimiento conceptual. Los conceptos están referidos a objetos, eventos o situaciones y se clasifican según su grado de exclusividad: conceptos supra ordinales, coordinados y subordinados. Cuando se vinculan dos o más conceptos forman una proposición relacionada por una palabra de enlace. Los conceptos son representados por círculos (nodos), y las palabras de enlace se expresan a través de líneas o flechas. Se puede deducir, que en los mapas conceptuales los conceptos y preposiciones se organizan formando jerarquías de diferente nivel de generalización o inclusión,

\section{Metodología}

Por metodología se entiende, los criterios metodológicos en los cuales se enmarca el desarrollo del estudio planteado, mencionando que se corresponde con una investigación de tipo descriptiva, según el criterio de Hernández, Fernández y Baptista (2006) establecen: "los estudios descriptivos buscan especificar las propiedades importantes de personas, grupos, comunidades 0 cualquier otro fenómeno que sea sometido a análisis" (pág. 122). Es decir, el estudio estuvo orientado según Chávez, (2007) "a la recolección de información relacionada con el estado real de las personas, situaciones 0 fenómenos, pero sustentadas en una revisión documental".

Entretanto, el diseño que se consideró adecuado es el de campo, sustentado en lo señalado por Palella y Martins (2010) estableciendo la investigación de campo "consiste en la recolección de datos directamente de la realidad donde ocurren los hechos, sin manipular o controlar variables.

Estudia los fenómenos sociales en su ambiente natural..." (pág. 68) En efecto, esta investigación fue de campo, porque la investigadora abordó la realidad, 


\section{Consideraciones Finales}

Las consideraciones finales de esta investigación son las que se muestran a continuación:

El uso de las estrategias instruccionales, sirven de herramientas fundamentales utilizadas por el docente para el logro del aprendizaje significativo en los estudiantes universitarios, ya que genera la comunicación asertiva, la reflexión, la flexibilidad en la conducta y a la comprensión de los procesos mentales.

Establece condiciones internas y externas que orientan el proceso enseñanza-aprendizaje hacia el logro de cambios positivos en el plano del pensamiento, el sentimiento y la acción, convirtiéndolos en personas exitosas.

Es importante, que los docentes vivan en constante revisión y planifique aplique y valore las estrategias didácticas para que incentiven al proceso lector que se encuentra inmerso en todas las actividades desarrolladas por las personas en todos los campos y áreas de desempeño, y sobre todo, en el escenario educacional, donde se forma integralmente al individuo

\section{Referencias}

Aguirre, R. (2008). Fomentar la Lectura y la Escritura en Estudiantes de Formación Docente. Acción Pedagógica, № 17, pp. 86-95.

Ausubel, D., y otros (1988). Pedagogía Educativa. México: McGraw-Hill.

Cifuentes, G. (2004). "Los Latinoamericanos no saben leer". Ultimas Noticias. pág. 2. Caracas.

Chávez, N. (2007). Introducción a la investigación Educativa. 3ra Edición Maracaibo. Zulia.

Díaz, B., y Hernández, G. (2002). Estrategias Docentes para un Aprendizaje Significativo. Segunda Edición. México: McGraw-Hill.

Dubois, M. (2002). "La Lectura en la Formación y la Actualización del Docente. Lectura y Vida. Número 23, pág. 15. 
Garza y Leventhal (2003). Aprender como aprender. Tercera Edición. México: Trillas.

González, M. (2003a). El Profesorado Universitario: Su Concepción y Formación como Modelo de Actuación Ética y Profesional. Universidad de La Habana. Cuba.

Hernández, Fernández y Baptista (2006). Metodología de la investigación. México. Editorial Trillas 2nda Edición McGraw-Hill.

López, L. (2008). “La Lectura un Hábito a Estimular”. El Universal, pág. $2 / 12$.

Mata, L. y Ávila, F. (2012). El Aprendizaje Significativo en la Educación de Calidad. Universidad Rafael Belloso Chacín. Recuperado de: http://virtual.urbe.edu/eventostexto/JNI/URB-044.pdf

Monereo, C. (2004). Estrategias de Enseñanza y Aprendizaje, Formación del Profesorado y Aplicación en la Escuela. Barcelona, España.

Palella, S. y Martins, F. (2010). Metodología de la Investigación Cuantitativa. Fondo Editorial de la Universidad Pedagógica Experimental Libertador. Caracas.

Puerta, M. (2000). Reflexiones sobre la Enseñanza de la Literatura: ¿Corazón o Razón? Educere. Año 4. Número 11, pág. 165.

Reimers, F. (2000). "Educación, Desigualdad y Opciones de Política en América Latina en el Siglo XIX". Revista Latinoamericana de Estudios Educativos, Vol. XXX, número 002, pp. 11-42.

Revilla, D. (2003). Manual de Estilos de Aprendizaje. Material Auto Instruccional para Docentes y Orientadores Educativos.

Sánchez, E. (2010). Estrategias de Lectura y Clases de Lectura. Comprensión Lectora. Recuperado de:

http://lecturaiush.blogspot.com/2010/03/estrategias-de-lectura-yclasesde.html 
Villamizar (2006). ¿Qué queremos decir con lectura ahora? Editorial Siglo XXI. México.

Vigotsky, (1987). Pensamiento y Lenguaje. Segunda Edición. Cuba: Pueblo y Educación. 


\section{Yajaira del Valle Cadenas Terán \\ e-mail: yajairaf@yahoo.com}

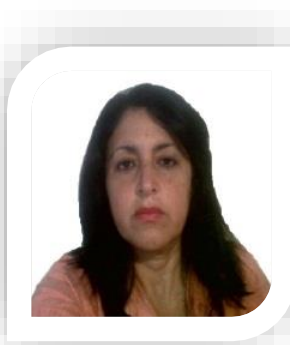

Nacida en Valera, Estado Trujillo, Venezuela. Actualmente cursa estudios de Doctorado en Ciencias de la Educación en la Universidad Nacional Experimental "Rafael María Baralt". Maestría en Administración de la Educación Básica Magíster Scientiarum 1994-1997; Diplomado en Cooperativismo y Gestión Comunitaria 2007 en el Instituto Universitario de Tecnología del Estado Trujillo; Curso de: Didáctica Especial en el Área de Castellano y Literatura, Matemática y Física y en el Área de Biología y Química 160 Horas 2010 (Diplomado) en el Liceo Bolivariano Antonio José Pacheco Estado Trujillo, convenio Cuba Venezuela. Experiencia Laboral actualmente Prof. Agregado de la Universidad Politécnica Territorial "Mario Briceño Iragorry".

El contenido de este manuscrito se difunde bajo una Licencia de Creative Commons ReconocimientoNoComercial-Compartirlgual 4.0 Internacional 